\title{
Tumor Uptake of Anti-CD20 Fabs Depends on Tumor Perfusion
}

\author{
Claudia Theresa Mendler*1,2, Annette Feuchtinger*3, Irina Heid*4, Michaela Aichler ${ }^{3}$, Calogero D'Alessandria ${ }^{1}$, \\ Sabine Pirsig ${ }^{1}$, Birgit Blechert ${ }^{1}$, Hans-Jürgen Wester ${ }^{5}$, Rickmer Braren ${ }^{4}$, Axel Walch ${ }^{3}$, Arne Skerra ${ }^{2}$, \\ and Markus Schwaiger ${ }^{1}$
}

${ }^{I}$ Nuklearmedizinische Klinik und Poliklinik, Klinikum rechts der Isar, Technische Universität München, München, Germany; ${ }^{2}$ Munich Center for Integrated Protein Science (CIPS-M) and Lehrstuhl für Biologische Chemie, Technische Universität München, Freising (Weihenstephan), Germany; ${ }^{3}$ Research Unit Analytical Pathology, Institute of Pathology, Helmholtz Zentrum München, Neuherberg, Germany; ${ }^{4}$ Institute of Radiology, Klinikum rechts der Isar, Technische Universität München, München, Germany; and

${ }^{5}$ Pharmaceutical Radiochemistry, Technische Universität München, Garching, Germany

Antibodies have become an established treatment modality in cancer therapy during the last decade. However, these treatments often suffer from an insufficient and heterogeneous response despite validated antigen or target receptor expression in the tumor. In fact, therapeutic success depends on both the presence of the tumor antigen and its accessibility by the antibody. In search of a suitable preclinical animal model to evaluate the mechanisms of tumor heterogeneity and hemodynamics, we characterized two exemplary non-Hodgkin lymphoma subtypes with comparable CD20 expression and metabolism, SUDHL-4 and Granta-519, using multimodal imaging techniques. Methods: To investigate in vivo biodistribution, two differently modified aCD20 antigen-binding fragments (Fab), prepared by PASylation with a 200-residue polypeptide tag comprising Pro, Ala, and Ser $\left(\mathrm{PAS}_{200}\right)$ and by fusion with an albumin-binding domain (ABD), were radiolabeled with ${ }^{125}$ and intravenously injected into immunocompromised mice bearing corresponding xenografts. Results: Validation with ${ }^{18} \mathrm{~F}-\mathrm{FDG}$ revealed a similar distribution in vital tumor tissue $1 \mathrm{~h}$ after injection. However, large differences in tumor uptake were observed when the CD20-specific radiotracers ${ }^{125}$ I-Fab-ABD and ${ }^{125}$ I-Fab$\mathrm{PAS}_{200}$ were applied (respective percentages injected dose per gram at $24 \mathrm{~h}$ after injection: 12.3 and 2.4 for Granta-519 vs. 5.8 and 1.2 for SUDHL-4). Three-dimensional light-sheet fluorescence microscopy with Cy5-Fab-PAS ${ }_{200}$ confirmed better tracer extravasation in the Granta-519 tumors. Moreover, dynamic contrast-enhanced (DCE) MRI revealed significantly reduced perfusion in the SUDHL-4 tumors. Conclusion: Tracer uptake was highly dependent on local tumor perfusion and Fab permeation in the SUDHL-4 and Granta-519 tumors. Thus, the SUDHL-4 xenograft offers an excellent model for investigating the influence of therapies affecting tumor angiogenesis.

Key Words: 3D light-sheet fluorescence microscopy; DCE MRI; Fab fragment; lymphoma; tumor perfusion

J Nucl Med 2016; 57:1971-1977

DOI: 10.2967/jnumed.116.176784

\footnotetext{
Received Apr. 13, 2016; revision accepted Jun. 21, 2016.

For correspondence or reprints contact: Claudia Theresa Mendler, Nuklearmedizinische Klinik und Poliklinik, Klinikum rechts der Isar der Technischen Universität München, Ismaninger Strasse 22, D-81675 München, Germany.

E-mail: claudia.mendler@wzw.tum.de

${ }^{*}$ Contributed equally to this work.

Published online Jul. 14, 2016.

COPYRIGHT (C) 2016 by the Society of Nuclear Medicine and Molecular Imaging, Inc.
}

$\mathbf{N}$ on-Hodgkin lymphomas (NHLs) are among of the most common malignancies, accounting for approximately $4 \%$ of all cancers. Most of them originate from B cells, which express the integral transmembrane protein CD20 at high levels. NHL subtypes are characterized by a distinct histopathologic anatomy and a complex and variable vascularity (1).

Antibody-based therapy, including radioimmunotherapy, is one of the most important strategies today for treating patients with NHLs and solid tumors (2). Recently, the accumulation and heterogeneity of ${ }^{111}$ In-ibritumomab tiuxetan and ${ }^{18}$ F-FDG uptake in tumors were analyzed and compared with the radioimmunotherapy response in 16 patients with B-cell NHL (3). It was found that the heterogeneity of intratumoral ${ }^{111} \mathrm{In}$-ibritumomab tiuxetan distribution, rather than the absolute level of antibody uptake, correlated with tumor response. Interestingly, no differences in heterogeneity were observed between responder and nonresponder groups when the small-molecule metabolic radiotracer ${ }^{18} \mathrm{~F}-\mathrm{FDG}$ was used.

Such studies clearly show that therapeutic success is highly influenced by the intratumoral access of the therapeutic antibody, a generally problematic feature of this class of high-molecularweight proteins. Especially in radioimmunotherapy, a nonuniform distribution within tumors appears to limit efficacy (4). Therefore, attempts are being made to search for diagnostic biomarkers to predict therapeutic outcome using histopathology (5) or perfusion measurements (1).

To our knowledge, there are no well-characterized preclinical xenograft models showing mutually similar tumor biology and target expression despite profound differences in tumor perfusion. Hence, the goal of this study was to compare and investigate two exemplary NHL subtypes, the mantle cell lymphoma cell line Granta-519 (6) and the diffuse large B-cell lymphoma cell line SUDHL-4 (7)-which exhibit a similar CD20 expression levelas subcutaneous human tumor xenograft models in immunocompromised mice using multimodal imaging.

\section{MATERIALS AND METHODS}

\section{Preparation and Radioiodination of aCD20 Fabs}

The CD20-specific Fab, derived from ofatumumab (2F2), was produced in Escherichia coli in two versions, fused either with an albumin-binding domain (ABD) or with a long conformationally disordered amino acid sequence consisting of 100 proline, alanine, and 
serine residues $\left(\mathrm{PAS}_{200}\right)$ and labeled with ${ }^{125} \mathrm{I}$ using the IODO-GEN method as previously described (8).

\section{Radiosynthesis of ${ }^{18}$ F-FDG}

${ }^{18} \mathrm{~F}-\mathrm{FDG}$ was produced by the Radiopharmacy Unit of Technische Universität München essentially as described before (9).

\section{In Vivo Studies}

SUDHL-4 and Granta-519 cells were grown in RPMI medium supplemented with $10 \%(\mathrm{v} / \mathrm{v})$ fetal bovine serum and $1 \mathrm{mM} \mathrm{L}$-glutamine. CB-17 SCID mice (Charles River Laboratories) were injected subcutaneously with $10 \times 10^{6}$ SUDHL- 4 cells or $5 \times 10^{6}$ Granta-519 cells suspended in $100 \mu \mathrm{L}$ of a $50 \%(\mathrm{v} / \mathrm{v})$ mixture of RPMI medium and Matrigel (Sigma-Aldrich). A maximum tumor size of approximately $800 \mathrm{~mm}^{3}$ was used for all experiments. For biodistribution studies, 5 mice were injected intravenously with $0.5 \mathrm{MBq}$ of ${ }^{125} \mathrm{I}$-Fab with a 200-residue polypeptide tag comprising Pro, Ala, and Ser $\left(\mathrm{PAS}_{200}\right)$ or ${ }^{125} \mathrm{I}-\mathrm{Fab}-\mathrm{ABD}$ as described before (8). All animal experiments complied with regulatory and institution guidelines and were approved by the local authorities (Regierung von Oberbayern, Germany; license 55.2-1-54-2532-87-11).

\section{Histology and Immunohistochemistry}

Excised specimens were fixed in $4 \%$ (w/v) neutrally buffered formalin, embedded in paraffin, and cut into $3-\mu \mathrm{m}$ slices. Immunohistochemical staining was performed on a Discovery XT automated stainer (Ventana Medical Systems) using the following antibodies: MIB1 (DCS Innovative Diagnostik-Systeme), CD31 (Abcam), CD20 (DakoCytomation), and Caspase 3 (Cell Signaling), with Discovery Universal (Ventana Medical Systems) as a secondary antibody. Signal was detected using the peroxidase-diaminobenzidine reaction (Ventana Medical Systems). Immunohistologic staining was quantified by image analysis using Developer XD2 software (Definiens) following a previously published procedure (10).

\section{Autoradiography}

Tumor sections $(10 \mu \mathrm{m})$ were incubated for $2 \mathrm{~h}\left({ }^{18} \mathrm{~F}\right)$ or $12 \mathrm{~h}\left({ }^{125} \mathrm{I}\right)$ on a Storage Phosphor Screen GP (Eastman Kodak Co.) in the dark. Distribution of radioactivity was subsequently quantified using a CR 35 Bio image plate scanner (Raytest Isotopenmessgeräte) with an internal resolution of $25 \mu \mathrm{m}$, followed by image analysis with AIDA software (Raytest Isotopenmessgeräte).

\section{Light-Sheet Fluorescence Microscopy}

Fab-PAS $200(2 \mathrm{mg} / \mathrm{mL})$ was coupled at a 5:1 molar ratio with Cy5-

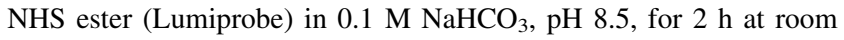
temperature. After removal of excess dye via a PD-10 column in phosphate-buffered saline, a labeling ratio of 1.6 fluorophores per Fab was determined via ultraviolet-visible spectroscopy.

CB-17 SCID mice were intravenously injected with $0.7 \mathrm{nmol}$ of Cy5-labeled Fab-PAS ${ }_{200}$ and, after 24 h, with 2 nmol of lectin from Bandeiraea (Griffonia) simplicifolia-isolectin I (L2380; SigmaAldrich) labeled with VivoTag-S 750 (PerkinElmer)-for specific vessel visualization-followed by incubation for $5 \mathrm{~min}$ before the animals were sacrificed. Explanted tumors were fixed in PaxGene (PreAnalytiX) as described previously (11) and then subjected to a chemical procedure of optical clearing (12).

Whole tumors were imaged with a 3-dimensional UltraMicroscope (LaVision BioTec). The specimens were illuminated on two sides by a planar light-sheet using a white-light laser (SuperK Extreme EXW-9; NKT Photonics). Lectin and Cy5-Fab-PAS 200 were excited at 740/35 nm and $640 / 30 \mathrm{~nm}$, respectively, whereas the emitting light was detected using 795/50 nm and 690/50 nm filters. Optical sections were recorded by moving the specimen chamber vertically at $5-\mu \mathrm{m}$ steps through the laser light-sheet. Three-dimensional reconstructions were obtained using ImspectorPro, version 5.0.1 (LaVision BioTec), and Vision4D (arivis).

\section{Dynamic Contrast-Enhanced (DCE) MRI}

The mice were anesthetized with isofluran, temperature was controlled, and the mice were placed prone on a 47-mm microscopy surface coil in the 1.5-T Achieva clinical scanner (Philips). An axial multislice T2-weighted turbo spin-echo sequence (resolution, $0.3 \times 0.3 \times 0.7 \mathrm{~mm}$; echo time, $90 \mathrm{~ms}$; repetition time, $>3 \mathrm{~s}$; number of signal averages, 8 ) covering the entire tumor was applied as the anatomic reference. DCE MRI experiments were performed using a previously described fast single-shot "look-locker"-based radial T1 mapping technique with the "golden cut" principle $(13,14)$. Dynamic T1 mapping of one axial slice positioned over the tumor and spinal muscles was performed every $5.7 \mathrm{~s}$ for a total of $1,075 \mathrm{~s}$ (resolution, $0.7 \times 0.7 \times 2 \mathrm{~mm}$; echo time, $5.7 \mathrm{~ms}$; repetition time, $12.6 \mathrm{~ms}$; radial profiles, 105; flip angle, $10^{\circ}$; acquisition time, $2.6 \mathrm{~s}$; pause time, $3.1 \mathrm{~s}$ ). Then, one $0.04 \mathrm{mmol} / \mathrm{kg}$ bolus of gadolinium-diethylenetriaminepentaacetic acid (DTPA) (Magnevist) was administered via the tail vein after $57 \mathrm{~s}$ of baseline measurement. DCE MRI data were analyzed using in-house software. Areas under the gadolinium-concentration curve for vital tumor and spinal muscle were computed using Prism 5 (GraphPad).

\section{Immunofluorescence}

CB-17 SCID mice were injected intravenously with $15 \mathrm{mg} / \mathrm{kg}$ of Hoechst33342 (H33342; Sigma Aldrich) 1 min before sacrifice. Tumor cryosections were stained with primary CD20-specific mouse monoclonal antibody (DakoCytomation) and CD31-specific rabbit polyclonal antibody (Abcam) using fluorescein-labeled goat antirabbit or Cy3-labeled goat antimouse IgG (both Invitrogen) as secondary antibodies. Fluorescence images were captured using an AxioImager Z1 (Zeiss) upright microscope system. Cy3-based signals were detected with HE DsRed Filter Set 43, fluorescein-based signals were detected with HE Green Fluorescent Protein Filter Set 38, and H33342 dye was detected with DAPI Filter Set 49 (all from Carl Zeiss).

\section{Diffusion Analysis with $\mathrm{H33342}$}

Images of entire tumor sections were acquired at 200-fold magnification with an AxioImager Z1 using the mosaic picture mode. For quantitative image analysis, regions of interest (vital tumor) were identified and analyzed using Developer XD2 (Definiens) as described previously (15). First, a specific rule set was defined to detect the CD31-stained vessel wall based on fluorescence layer, intensity, shape, and neighborhood. Next, a distance map algorithm was applied to define distance ranges surrounding the vessels with a stepwise increasing distance of $0-25 \mu \mathrm{m}$ up to $175-200 \mu \mathrm{m}$. Finally, the overall mean $\mathrm{H} 33342$ intensity was calculated for each vessel and distance range.

\section{Statistics}

For comparison between two groups, the Student $t$ test for unpaired data was used. $P$ values of less than 0.05 were considered statistically significant.

\section{RESULTS}

\section{Characterization of SUDHL-4 and Granta-519 Tumors}

The tumor morphologies of the two distinct NHL cell lines Granta-519 and SUDHL-4 were investigated with regard to in vivo biodistribution and autoradiography in immunocompromised mice using the metabolic radiolabel ${ }^{18} \mathrm{~F}-\mathrm{FDG}$ and the radioiodinated $\alpha \mathrm{CD} 20$ tracers ${ }^{125} \mathrm{I}-\mathrm{Fab}-\mathrm{PAS}_{200}$ and ${ }^{125} \mathrm{I}-\mathrm{Fab}-\mathrm{ABD} .{ }^{18} \mathrm{~F}-\mathrm{FDG}$ autoradiography revealed a homogeneous distribution pattern in both xenograft models, thus verifying metabolic activity and tumor viability (Fig. 1A). Also, the two versions of the CD20-specific Fab, both engineered for extended plasma half-life using either PASylation technology (XL-protein $\mathrm{GmbH}$ ) or an ABD, showed 


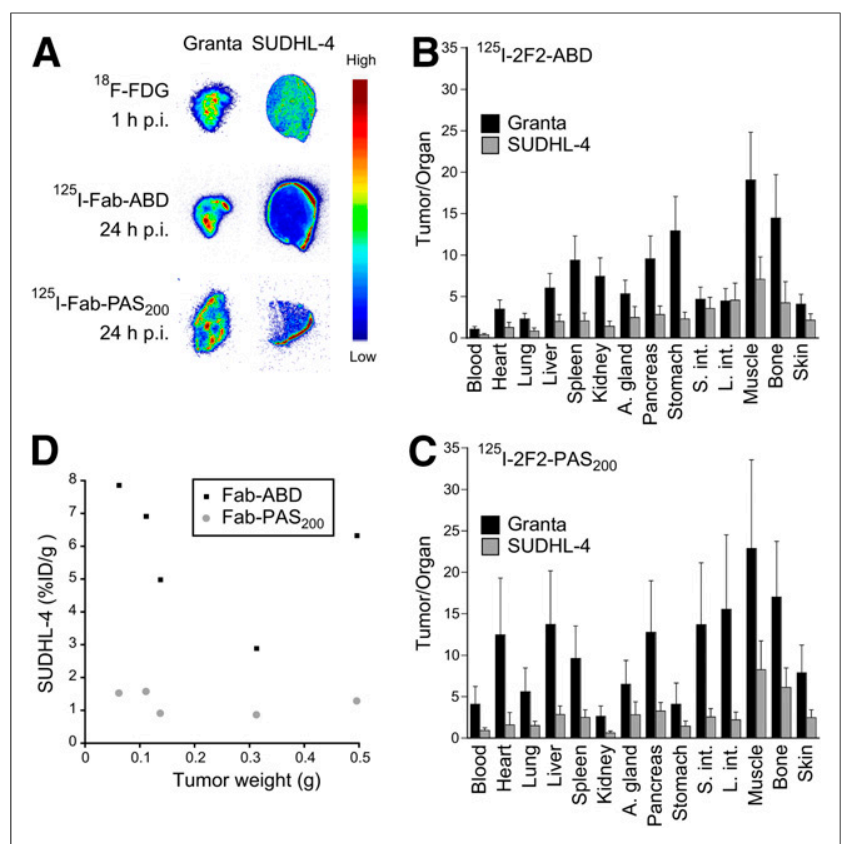

FIGURE 1. Autoradiography and biodistribution studies on Granta519 and SUDHL-4 tumors. (A) Autoradiography of tumor sections from immunocompromised mice was performed $24 \mathrm{~h}$ after injection of ${ }^{125} \mathrm{I}-\mathrm{Fab}-\mathrm{ABD}$ or ${ }^{125 \mathrm{I}-\mathrm{Fab}-\mathrm{PAS}}{ }_{200}$ or $1 \mathrm{~h}$ after injection of ${ }^{18} \mathrm{~F}-\mathrm{FDG}$. ( $B$ and $C$ ) Biodistribution studies of Fab-ABD and Fab-PAS 200 labeled with ${ }^{125} \mathrm{I}$ were conducted $24 \mathrm{~h}$ after injection. Tumor-to-organ ratios are plotted for both aCD20 Fabs (mean $\pm \mathrm{SD} ; n=5$ ). (D) No strong correlation between tumor uptake and tumor weight is found for SUDHL-4 tumors $24 \mathrm{~h}$ after injection, irrespective of the Fab radiotracer used (Pearson coefficient of -0.32 for Fab-PAS 200 and -0.36 for Fab-ABD).

strong, though heterogeneous, distribution in Granta-519 tumors $24 \mathrm{~h}$ after injection (Fig. 1A). In contrast, the SUDHL-4 tumors exhibited an unusual rim of concentrated tracer signal with minimal radioactivity in the tumor lumen.

These findings were confirmed by biodistribution experiments, which indicated strong tumor accumulation $24 \mathrm{~h}$ after injection for Fab-ABD and Fab-PAS 200 (respective percentages injected dose per gram: 12.3 and 2.4) and high tumor-to-organ ratios in the Granta-519 tumors (Supplemental Table 1; supplemental materials are available at http://jnm.snmjournals.org). However, drastically reduced tumor uptake, by a factor of around 2 for Fab-ABD and Fab$\mathrm{PAS}_{200}$, was observed for the SUDHL-4 tumors as also indicated by low tumor-to-organ ratios (Figs. 1B and 1C). Nevertheless, the tracer distribution in the organs was similar for both models. In addition, no correlation between tracer uptake and tumor size, typically in the range of $100-800 \mathrm{~mm}^{2}$, was seen (Fig. 1D).

For efficient tumor uptake and tracer distribution, the expression pattern of the target antigen CD20 is crucial. In agreement with previously published data, a high CD20 expression for both NHL cell lines was verified using fluorescence-activated cell sorting analysis (data not shown) before tumor inoculation (16). Also, immunohistochemical staining of explanted tumors showed high CD20 expression, with $100 \%$ of the cells being positive on all slides (Fig. 2A).

Apoptosis and proliferation are further factors that can influence tracer distribution and, thus, were here investigated (Fig. 2A). Immunohistochemical analysis of activated caspase 3 clearly revealed absence of large apoptotic areas, with less than $5 \%$ of the cells staining positively for total caspase 3 . Tumor viability was further confirmed by hematoxylin and eosin staining of SUDHL-4 tumors with a size of $800 \mathrm{~mm}^{3}$ (Fig. 2B). Notably, according to the Mib1 marker, a significantly higher proliferation rate was seen for the Granta-519 tumors $(P=0.01)$ (Fig. 2C).

Finally, both xenograft models showed a homogeneously distributed vasculature, with CD31-stained vessels in the tumor center and in the periphery (Fig. 2A). However, quantification of vessels revealed that vascularization was significantly higher, by a factor of around 2, in the Granta-519 tumors (Fig. 2D).

\section{Vessel Architecture, Tumor Perfusion, Microvascular Permeability, and Tissue Penetration}

Cy5-labeled Fab-PAS 200 and the $B$. (G.) simplicifolia-isolectin I conjugated with the near-infrared fluorochrome VivoTag-S 750, as a specific vessel marker, were injected intravenously into both xenograft models to correlate the distribution pattern of the Fab within the 3-dimensional vessel structure via light-sheet fluorescence microscopy (Fig. 3A). Lectin staining revealed good vascularization across the entire tumors. Notably, in the Granta-519 tumor a heterogeneous distribution of the fluorescently labeled Fab-PAS ${ }_{200}$ was found, similar to that observed with the radiolabeled Fab using autoradiography. In contrast, SUDHL-4 tumors showed a rimlike distribution pattern, again as seen using autoradiography. When the images obtained for the Fab and the lectin

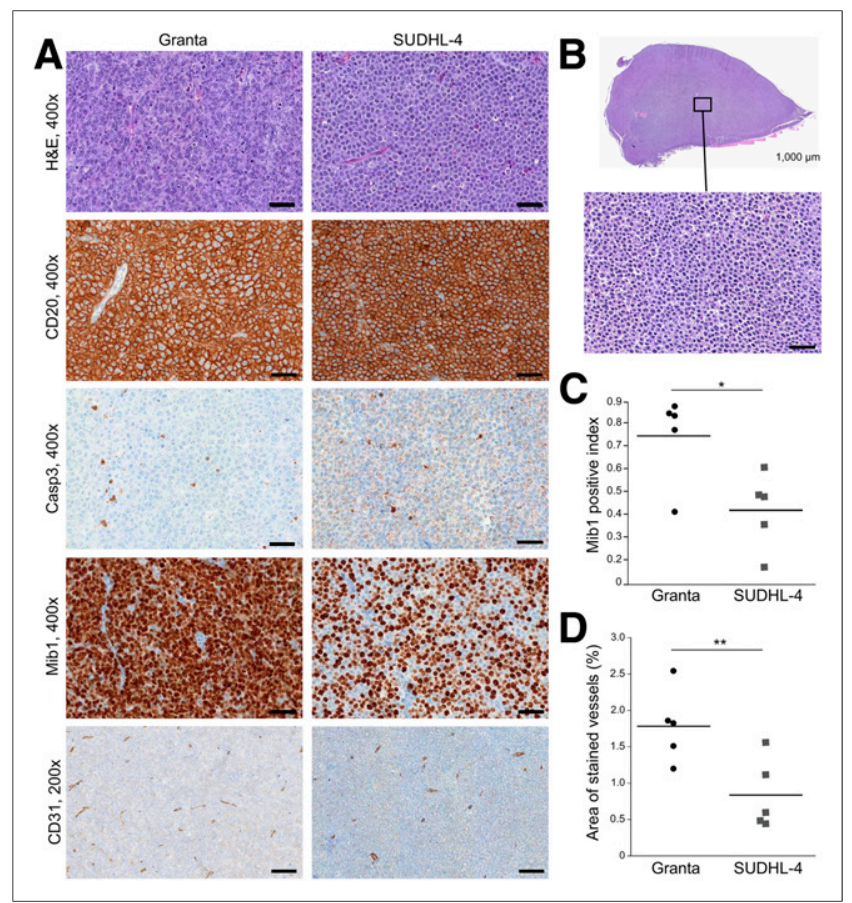

FIGURE 2. Histologic and immunohistochemical characterization of Granta-519 and SUDHL-4 tumors. (A) Explanted tumors were stained with hematoxylin and eosin (H\&E) or immunostained for the target antigen CD20, apoptosis marker caspase 3 (Casp3), proliferation marker Mib1, or endothelial vessel marker CD31 (scale bar, $50 \mu \mathrm{m}$ ). (B) Example is shown of H\&E-stained SUDHL-4 tumor comprising an area of $6 \times$ $10 \mathrm{~mm}$. (C) Quantification of Mib1 staining reveals significantly stronger proliferation for Granta-519 tumors than for SUDHL-4 tumors $(P=0.01)$. (D) Quantification of vessel area $(n=5)$ reveals significantly higher values for Granta-519 tumors than for SUDHL-4 tumors $(P<0.01)$. 

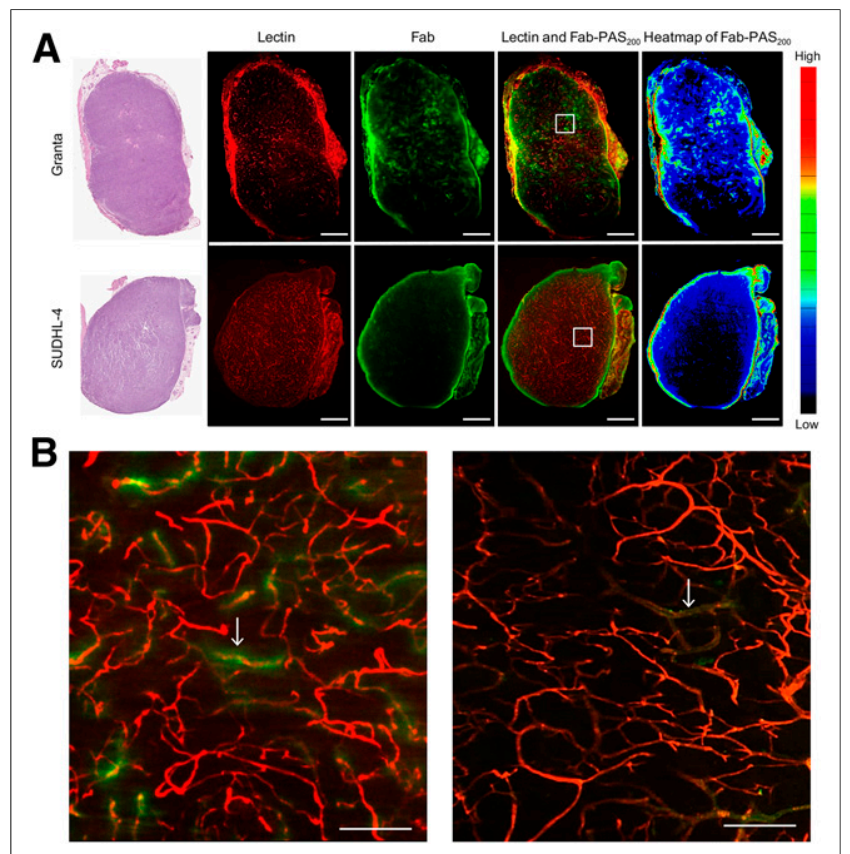

FIGURE 3. Visualization of vessels and aCD20 Cy5-Fab-PAS 200 distribution in Granta-519 and SUDHL-4 tumors. Both xenograft models were stained in vivo with fluorescence-labeled aCD20 Fab-PAS $200(24 \mathrm{~h}$ before sacrifice) and fluorescence-labeled $B$. (G.) simplicifolia-isolectin 1 for vessels ( 5 min before sacrifice) and imaged by light-sheet fluorescence microscopy. (A) Both xenograft models show extensive vascularization (red) throughout. Heterogeneous penetration and distribution of labeled Fab-PAS 200 (green) is observed across entire Granta-519 tumor, whereas for the SUDHL-4 tumor nearly no Fab-PAS 200 outside vessels is detected. Heat map visualization confirms this different distribution pattern of Granta519 and SUDHL-4 tumors (scale bar, 1,500 $\mu \mathrm{m}$ ). (B) Different localization of labeled Fab-PAS ${ }_{200}$ (green) in relation to lectin-labeled vessels (red) is clearly visible in magnified images of the maximum-intensity projection of 25 virtual single optical slices. Granta-519 tumor shows perivascular distribution of Fab-PAS ${ }_{200}$, whereas SUDHL-4 tumor shows confinement of Fab-PAS 200 to intravascular space (arrows).

were merged, localization of the Fab only inside the vessels was observed for the SUDHL-4 tumors whereas the Fab was clearly detected in the perivascular space for the Granta-519 tumors (Fig. 3B). However, not all vessels in the Granta-519 tumors seemed permeable for Cy5-Fab-PAS 200 (Supplemental Videos 1-5).

Next, noninvasive perfusion experiments using DCE MRI were performed on CB-17 SCID mice bearing Granta-519 and SUDHL4 tumors. The time-dependent concentration curves detected after a bolus injection of the low-molecular-weight contrast agent gadolinium-DTPA revealed two major components: first, active perfusion of the tissue based on the arterial input function, which is related to blood flow, and second, passive diffusion of gadolinium-DTPA into and out of the extravascular interstitial space, which predominantly depends on vessel permeability and surface area (17). A gadolinium-DTPA T1 map obtained before and $28.5 \mathrm{~s}$ after gadolinium-DTPA injection (Fig. 4A) indicated a higher intratumoral gadolinium-DTPA concentration in Granta519 than in SUDHL-4, in line with the radiotracer distribution experiments. All other organs, including muscles, showed a similar wash-in pattern independent of the xenograft model. Although both tumor models displayed high interindividual differences, the Granta-519 tumors revealed a consistently higher wash-in pattern according to the gadolinium-DTPA concentration-time curves than the SUDHL-4 tumors (Fig. 4B). Consequently, the tumorto-muscle ratios of the calculated areas under the curve revealed significantly lower perfusion in SUDHL-4 tumors (Supplemental Fig. 1).

Tumor vessel permeability was further analyzed using the fluorescent perfusion dye H33342, which intercalates into cell nuclei adjacent to permeable blood vessels. For simultaneous detection, two consecutive cryosections of animals treated with the radiolabeled Fab-ABD or Fab-PAS 200 and H33342 were analyzed via autoradiography and immunofluorescence, respectively (Fig. 5A). A pronounced heterogeneous distribution of $\mathrm{H} 33324$ was observed, but there was no correlation between the distribution of the Fab and that of H33342 (Fig. 5B).

Interestingly, comparison of $\mathrm{H} 33342$ penetration into the surrounding tumor tissue revealed different patterns: a broad uniform spreading of the perfusion dye was detected in the Granta-519 tumors, whereas in the SUDHL-4 tumors it was merely the cells near the tumor vessel that were stained (Fig. 5A). To quantify H33342 tissue penetration, image analysis via distance maps was applied and the H33342 intensity was calculated inside vessels and in the surrounding tumor tissue. Almost constant H33342 intensity was detected across the Granta-519 tumors within a distance of $200 \mu \mathrm{m}$ from a vessel, whereas SUDHL-4 tumors revealed a rapid decline in dye intensity with increasing distance from a vessel (Fig. 5C).

\section{DISCUSSION}

Tumor heterogeneity is one of the main challenges during clinical antibody diagnosis and therapy. Despite prevalent target expression, subgroups of patients do not properly respond to antibody treatment, and the uneven distribution of most antibodies in tumor tissue, including metastases, leaves whole tumor areas or

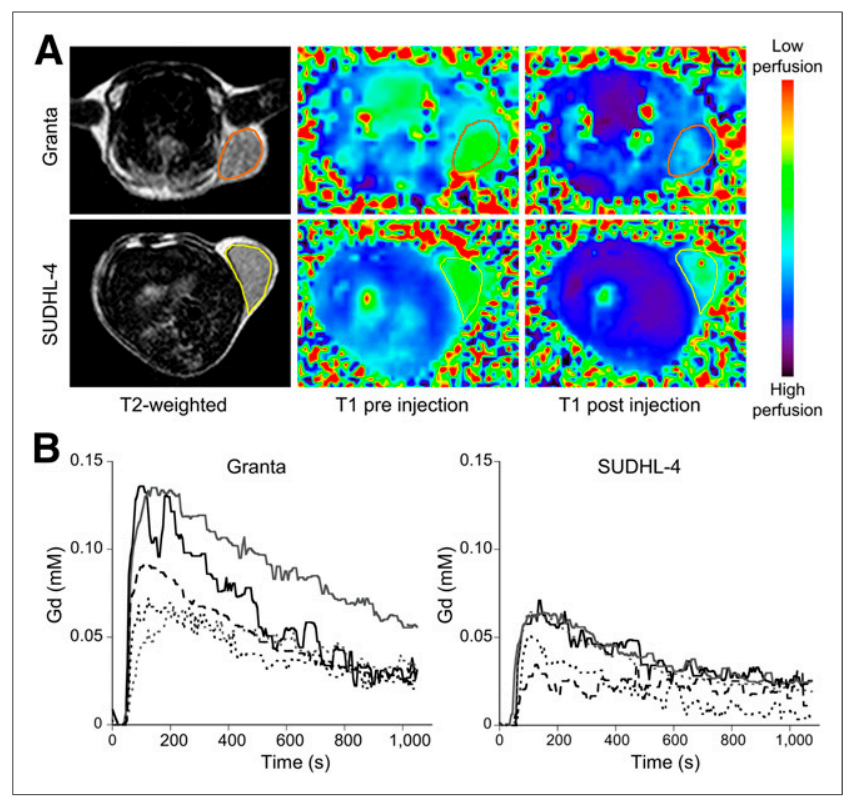

FIGURE 4. DCE MRI perfusion study of NHL xenograft models. (A) T2weighted anatomic images are shown of an example Granta-519 tumor and an example SUDHL-4 tumor and their corresponding gadoliniumDTPA T1-weighted maps with color scale. (B) Gadolinium-concentration curves show higher wash-in pattern in individual Granta-519 tumors than in SUDHL-4 tumors despite considerable interindividual variation. 


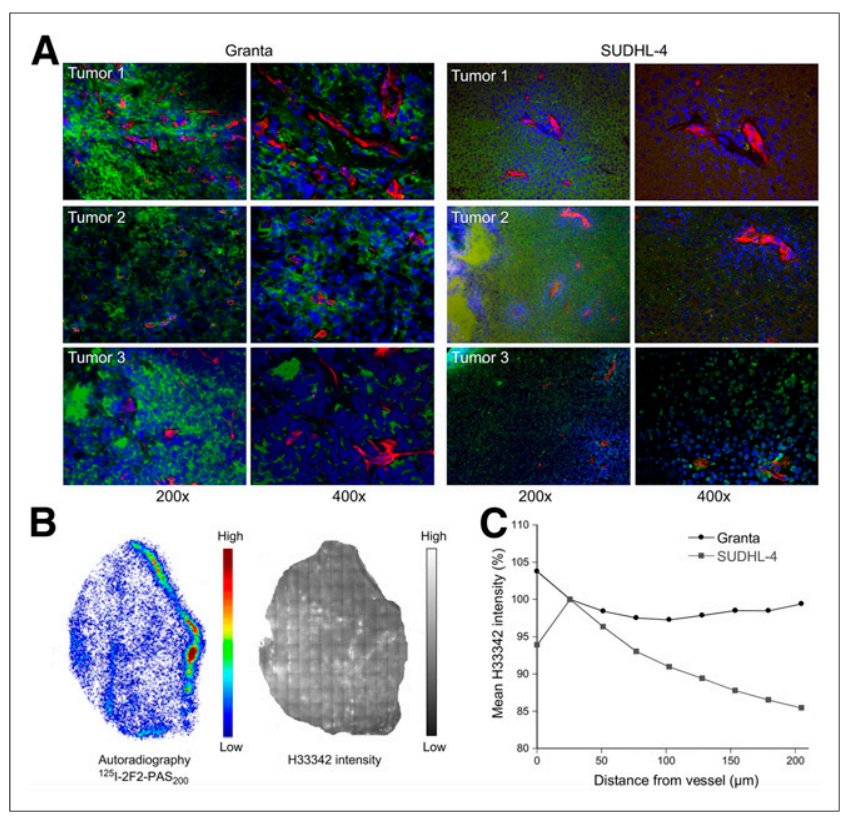

FIGURE 5. H33342 perfusion and penetration in Granta-519 and SUDHL-4 tumors. (A) Perfusion dye H33342 was injected $24 \mathrm{~h}$ after radioiodinated Fab-ABD or Fab-PAS ${ }_{200}$ and 1 min before mice were sacrificed. Afterward, CD20 (green) and CD31 (red) were detected in tumor cryosections using immunofluorescence. H33342 perfusion of 3 different SUDHL-4 and Granta-519 tumors is represented in blue. (B) Autoradiography and corresponding H33342 intensity is shown for an example SUDHL-4 tumor. (C) Mean H33342 penetration into tissue was quantified using distance maps based on CD31-positive vessels, with distance of zero equivalent to vessel lumen. $\mathrm{H} 33342$ intensity was normalized with regard to first distance value $(25 \mu \mathrm{m})$ after vessel. $2 \mathrm{~F} 2=\mathrm{CD} 20$-specific Fab, derived from ofatumumab.

lesions untargeted. Numerous clinical and experimental studies have shown that tumor perfusion has a strong impact on both radiotracer uptake and drug delivery. Early detection of changes in tumor permeability and perfusion during treatment would therefore be of great benefit for response prediction. To study the underlying mechanisms of tumor heterogeneity and the role of tumor permeability and perfusion, a well-defined preclinical animal model is highly desired. To this end, we characterized two NHL xenograft models, Granta-519 and SUDHL-4, with apparently similar tumor biology regarding morphology, metabolic activity, and CD20 target expression but overt differences in perfusion and hemodynamic properties.

Similar autoradiography distribution patterns were found for the small-molecule nontargeted radioactive tracer ${ }^{18} \mathrm{~F}-\mathrm{FDG}$ in both xenograft models. These result agree with a previous study that investigated SUDHL-4 tumors using ${ }^{18} \mathrm{~F}-\mathrm{FDG}$ and the well-established small-molecule metabolic tracer $3^{\prime}$-deoxy- $3^{\prime}-{ }^{18}$ F-fluorothymidine (7). Likewise, comprehensive investigation of Granta-519 tumors using the CD20-targeting radiolabeled antibody Fab fragments applied here $\left(\mathrm{Fab}-\mathrm{PAS}_{200}\right.$ and Fab-ABD) revealed strong uptake, similar to an analogous Fab tracer derived from trastuzumab that was previously investigated in a HER2-positive xenograft model (8).

In contrast, almost no specific tumor uptake and a highly unusual rimlike accumulation pattern were observed in SUDHL-4 tumors for both PASylated and ABD-fused $\alpha \mathrm{CD} 20$ Fab versions, and a lower intratumoral gadolinium-DTPA concentration was de- tected by DCE MRI analysis. Furthermore, investigation of tracer localization at a cellular resolution level using light-sheet fluorescence microscopy revealed broad transcapillary diffusion of the tracer into the surrounding tissue of Granta-519 tumors, whereas the Fab tracer remained mainly intravascular in the SUDHL-4 tumors. H33342 perfusion studies confirmed these results, showing reduced extravasation and tissue penetration of the fluorescent dye in the SUDHL-4 tumors. Finally, the superior perfusion properties of the Granta-519 tumor were in line with the immunohistochemical quantification of CD31-positive tumor vessels, which indicated a significantly higher density of tumor vasculature in Granta-519 than in SUDHL-4.

The distinct behavior of the CD20-positive SUDHL-4 and Granta-519 tumors with respect to the various tracers investigated here can be explained in part by their differing biomolecular properties. In comparison to the rapidly excreted small-molecule imaging compounds ${ }^{18} \mathrm{~F}-\mathrm{FDG}$, gadolinium-DTPA, and $\mathrm{H} 33342$, with a molecular size of less than $1 \mathrm{kDa}$, both versions of the recombinant $\alpha \mathrm{CD} 20$ antibody fragment, Fab-ABD and Fab-PAS ${ }_{200}$, exhibit much larger apparent molecular sizes of $40 \mathrm{kDa}(\geq 100 \mathrm{kDa}$ after association with albumin) and $219 \mathrm{kDa}$, respectively, and, consequently, much longer plasma half-lives of 29 and $5 \mathrm{~h}$, although having the same antigen affinity (18). Low-molecular-weight compounds usually can rapidly cross the capillary wall, whereas large molecules such as monoclonal antibodies show slower transcapillary and interstitial transport. Therefore, the influence of tumor vessel architecture and physiology has a greater impact on macromolecular tracers such as antibodies and Fab fragments.

In general, the existence of different types of blood vessels is known from the literature. In healthy tissue, a regularly patterned and functional vascularization is found, with a normal vessel wall and endothelium. These semipermeable vessels, with a pore size of $5 \mathrm{~nm}$, allow the efflux of both small molecules and proteins up to around $60 \mathrm{kDa}$ but hinder transcapillary transport of larger proteins, particularly antibodies (19). For tumors, on the other hand, vessels with a leaky, tortuous, dilated, and saccular phenotype and a random pattern of interconnections are described, most likely caused by an imbalance of pro- and antiangiogenic signaling $(20,21)$. This perforated phenotype should permit a similar distribution of macromolecular tracers and small-molecule reagents outside the tumor vessels. Indeed, in the Granta-519 tumors we have seen high extravasation for the Fab and all 3 smallmolecule compounds. Nevertheless, some vessels did not show this leaky phenotype and were impermeable for the $\alpha \mathrm{CD} 20 \mathrm{Fab}$ as observed by light-sheet fluorescence microscopy.

Interestingly, the SUDHL-4 tumor did not match this phenotype, since accumulation of both the Fab-ABD and the smallmolecule gadolinium-DTPA and H33342 was clearly reduced. Hence, there seems to be a second type of distorted tumor vasculature that is characterized by a more closed, less permeable vasculature. This prevents extravasation of macromolecules and also some small molecules (depending on size, hydrophobicity, or charge), whereas low-molecular-weight metabolites, in particular amino acids and glucose, may still reach the tumor. In fact, such a less permeable vasculature is in accordance with the known low or heterogeneous tumor penetration of antibodies or drugs previously observed in certain other xenograft models (22) and, even more importantly, in patients (3).

Interstitial hypertension is another factor in tumor physiology that may play a role in tumor targeting and tissue penetration $(19,22)$ and, in principle, could offer an explanation for the low 
tracer uptake seen here in the SUDHL-4 tumors. Indeed, in the biomedical literature high interstitial fluid pressure is assumed to prevent efficient tumor penetration of reagents for imaging and therapy (23). However, although a positive pressure within the tumor tissue would clearly counteract convective molecular transport, it is a misconception that interstitial fluid pressure should slow down intratumor molecular diffusion.

Interstitial fluid pressure is likely caused by local colloid osmotic pressure due to a higher concentration of macromolecules such as polypeptides or proteoglycans in the tumor interstitium. Notably, this phenomenon somewhat contradicts the concept of generally permeable tumor vasculature mentioned above and its consequences for macromolecular accumulation discussed further below. Even in biologic systems, Newton's law of actio est reactio applies, which means that under circumstances of tissue homeostasis the difference in osmotic pressure between capillary lumen and (tumor) interstitium must equal the static pressure between both compartments. In contrast, diffusion is driven by individual concentration differences-that is, the chemical potential for each molecular species - which is only marginally affected by static pressure (at least at the moderate values prevailing in a living organism).

Apart from that, there are a couple of other aspects that govern efficient tumor targeting, especially for protein-based tracers or drugs (24). First, there is the general aspect of vascular permeability already discussed above. Importantly, most tumors lack lymph drainage, which prevents a convective force that drives permeation of macromolecules through healthy tissues. However, the defective and leaky blood vessels seen in many tumors are considered to cause the so-called enhanced permeability and retention effect. This phenomenon describes the extensive leakage of blood plasma components, in particular large proteins and other macromolecules larger than $40 \mathrm{kDa}$, into the tumor tissue and their nonspecific accumulation there (25). Although the enhanced permeability and retention effect is in fact assumed to promote tumor targeting by antibodies (and liposomes, viruslike particles, and so forth), it does not guarantee proper distribution within the tumor tissue, beyond the capillary vessel.

On the other hand, the most obvious diffusional barrier within the tumor interstitium is the extracellular matrix-especially in the context of a usually crowded tumor parenchyma-which hampers intratumoral distribution of macromolecules in a size-dependent manner (26). Although this aspect deserves more detailed investigation, it has become clear that not only glycosaminoglycans but also various collagens form part of the gellike structure that causes an interstitial transport resistance and slows down diffusion of larger molecules. Taken together, the situation is complex, and welldefined models are needed. The two distinct xenograft models characterized in the present study should prove useful for dissecting the effects of tumor vessel permeability, extracellular matrix composition, and, possibly, interstitial fluid pressure in dedicated experimental setups.

In the clinical situation, detection and treatment of tumors with a complex and heterogeneous physiology is difficult, and patients often face inefficient diagnostic or therapeutic efficacy. Although, for example, the combination of the CD20-specific antibody rituximab with standard chemotherapy results in higher overall survival and complete response rates in many subtypes of NHL, a significant proportion of patients relapse with incurable disease (27). Therefore, assessment of tumor perfusion and permeability in addition to common ${ }^{18}$ F-FDG PET staging could considerably improve tumor diagnosis and, eventually, therapy. Indeed, a recently published clinical study showed that adverse findings on perfusion and diffusion MRI correlated with adverse prognosis in immunocompetent patients with primary central nervous system lymphoma (28).

On the basis of our present investigation, the SUDHL-4 xenograft is a suitable preclinical model for investigating the mechanisms of hampered tumor perfusion and permeability for protein therapeutics or tracers, including strategies for tumor vessel normalization or other approaches to improve extravasation and tissue distribution. Several studies have shown that normalization of leaky, abnormal tumor vessels is possible by applying antiangiogenic therapies such as bevacizumab (29). One study found a decrease in interstitial fluid pressure by more than $70 \%$ and augmented tumor radiation response in two different mouse models bearing human tumor xenografts after anti-vascular endothelial growth factor treatment (30). During this normalization process, immature vessels were pruned and the integrity and function of the vasculature was improved by enhancing the perivascular cell and basement membrane coverage, eventually resulting in a deeper penetration of molecules into the tumor tissue. Another study demonstrated improvement of specific antibody uptake by targeting the tumor stromal microenvironment with hyaluronidase, thus altering the tumor extracellular matrix (31). Consequently, the SUDHL-4 xenograft offers a model to study alternative CD20-specific tracers with smaller size and faster diffusion than antibodies, such as Fab fragments, anticalins, Affibody molecules, nanobodies, or even peptides.

\section{CONCLUSION}

Tracer uptake was highly dependent on local tumor perfusion and Fab permeation in the SUDHL-4 and Granta-519 tumors. Thus, the SUDHL-4 xenograft offers an excellent model for investigating the influence of therapies affecting tumor angiogenesis.

\section{DISCLOSURE}

The costs of publication of this article were defrayed in part by the payment of page charges. Therefore, and solely to indicate this fact, this article is hereby marked "advertisement" in accordance with 18 USC section 1734. This study was supported by ERC grant MUMI (ERC-2011-ADG_20110310) and by the Deutsche Forschungsgemeinschaft Collaborative Research Centre (SFB 824). Arne Skerra is a shareholder of XL-protein GmbH. No other potential conflict of interest relevant to this article was reported.

\section{ACKNOWLEDGMENTS}

We thank Prof. Dr. Buck and Dr. Keller for providing the tumor cell lines SUDHL-4 and Granta-519.

\section{REFERENCES}

1. Spira D, Adam P, Linder C, et al. Perfusion and flow extraction product as potential discriminators in untreated follicular and diffuse large B cell lymphomas using volume perfusion CT with attempt at histopathologic explanation. AJR. 2012;198:1239-1246.

2. Scott AM, Wolchok JD, Old LJ. Antibody therapy of cancer. Nat Rev Cancer. 2012;12:278-287.

3. Hanaoka K, Hosono M, Tatsumi Y, et al. Heterogeneity of intratumoral ${ }^{111} \mathrm{In}$ ibritumomab tiuxetan and ${ }^{18} \mathrm{~F}-\mathrm{FDG}$ distribution in association with therapeutic response in radioimmunotherapy for B-cell non-Hodgkin's lymphoma. EJNMMI Res. 2015;5:10.

4. Kalogianni E, Flux GD, Malaroda A. The use of BED and EUD concepts in heterogeneous radioactivity distributions on a multicellular scale for targeted radionuclide therapy. Cancer Biother Radiopharm. 2007;22:143-150. 
5. Ruan J, Hyjek E, Kermani P, et al. Magnitude of stromal hemangiogenesis correlates with histologic subtype of non-Hodgkin's lymphoma. Clin Cancer Res. 2006;12:5622-5631.

6. Gopal AK, Press OW, Wilbur SM, Maloney DG, Pagel JM. Rituximab blocks binding of radiolabeled anti-CD20 antibodies (Ab) but not radiolabeled antiCD45 Ab. Blood. 2008;112:830-835.

7. Graf N, Herrmann $K$, Numberger B, et al. $\left[{ }^{18} \mathrm{~F}\right] \mathrm{FLT}$ is superior to $\left[{ }^{18} \mathrm{~F}\right] \mathrm{FDG}$ for predicting early response to antiproliferative treatment in high-grade lymphoma in a dose-dependent manner. Eur J Nucl Med Mol Imaging. 2013;40:34-43.

8. Mendler CT, Friedrich L, Laitinen I, et al. High contrast tumor imaging with radio-labeled antibody $\mathrm{Fab}$ fragments tailored for optimized pharmacokinetics via PASylation. MAbs. 2015;7:96-109.

9. Hamacher K, Coenen HH, Stöcklin G. Efficient stereospecific synthesis of nocarrier-added 2-[ $\left.{ }^{18} \mathrm{~F}\right]$-fluoro-2-deoxy-D-glucose using aminopolyether supported nucleophilic substitution. J Nucl Med. 1986;27:235-238.

10. Feuchtinger A, Stiehler T, Jütting U, et al. Image analysis of immunohistochemistry is superior to visual scoring as shown for patient outcome of esophageal adenocarcinoma. Histochem Cell Biol. 2015;143:1-9.

11. Curti BD, Urba WJ, Alvord WG, et al. Interstitial pressure of subcutaneous nodules in melanoma and lymphoma patients: changes during treatment. Cancer Res. 1993;53:2204-2207.

12. Dobosz M. Multispectral fluorescence ultramicroscopy: three-dimensional visualization and automatic quantification of tumor morphology, drug penetration, and antiangiogenic treatment response. Neoplasia. 2014;16:1-13.

13. Steingoetter A, Svensson J, Kosanke Y, et al. Reference region-based pharmacokinetic modeling in quantitative dynamic contrast-enhanced MRI allows robust treatment monitoring in a rat liver tumor model despite cardiovascular changes. Magn Reson Med. 2011;65:229-238.

14. Winkelmann S, Schaeffter T, Koehler T, Eggers H, Doessel O. An optimal radial profile order based on the Golden Ratio for time-resolved MRI. IEEE Trans Med Imaging. 2007;26:68-76.

15. Huber K, Feuchtinger A, Borgmann DM, et al. Novel approach of MALDI drug imaging, immunohistochemistry, and digital image analysis for drug distribution studies in tissues. Anal Chem. 2014;86:10568-10575.

16. Meyer zum Büschenfelde C, Feuerstacke Y, Gotze KS, Scholze K, Peschel C. GM1 expression of non-Hodgkin's lymphoma determines susceptibility to rituximab treatment. Cancer Res. 2008;68:5414-5422.
17. Gillies RJ, Schornack PA, Secomb TW, Raghunand N. Causes and effects of heterogeneous perfusion in tumors. Neoplasia. 1999;1:197-207.

18. Schlapschy M, Binder U, Börger C, et al. PASylation: a biological alternative to PEGylation for extending the plasma half-life of pharmaceutically active proteins. Protein Eng Des Sel. 2013;26:489-501.

19. Carmeliet P, Jain RK. Molecular mechanisms and clinical applications of angiogenesis. Nature. 2011;473:298-307.

20. Carmeliet P, Jain RK. Principles and mechanisms of vessel normalization for cancer and other angiogenic diseases. Nat Rev Drug Discov. 2011;10:417-427.

21. Azzi S, Hebda JK, Gavard J. Vascular permeability and drug delivery in cancers. Front Oncol. 2013;3:211.

22. Heine M, Freund B, Nielsen $\mathrm{P}$, et al. High interstitial fluid pressure is associated with low tumour penetration of diagnostic monoclonal antibodies applied for molecular imaging purposes. PLoS One. 2012;7:e36258.

23. Heldin $\mathrm{CH}$, Rubin K, Pietras K, Östman A. High interstitial fluid pressure: an obstacle in cancer therapy. Nat Rev Cancer. 2004;4:806-813.

24. Al-Abd AM, Aljehani ZK, Gazzaz RW, et al. Pharmacokinetic strategies to improve drug penetration and entrapment within solid tumors. J Control Release. 2015;219:269-277.

25. Iyer AK, Khaled G, Fang J, Maeda H. Exploiting the enhanced permeability and retention effect for tumor targeting. Drug Discov Today. 2006;11:812-818.

26. Netti PA, Berk DA, Swartz MA, Grodzinsky AJ, Jain RK. Role of extracellular matrix assembly in interstitial transport in solid tumors. Cancer Res. 2000;60:2497-2503.

27. Fisher RI. Overview of non-Hodgkin's lymphoma: biology, staging, and treatment. Semin Oncol. 2003;30:3-9.

28. Valles FE, Perez-Valles CL, Regalado S, Barajas RF, Rubenstein JL, Cha S. Combined diffusion and perfusion MR imaging as biomarkers of prognosis in immunocompetent patients with primary central nervous system lymphoma. Am J Neuroradiol. 2013;34:35-40.

29. Muhsin M, Graham J, Kirkpatrick P. Bevacizumab. Nat Rev Drug Discov. 2004;3:995-996.

30. Lee CG, Heijn M, di Tomaso E, et al. Anti-vascular endothelial growth factor treatment augments tumor radiation response under normoxic or hypoxic conditions. Cancer Res. 2000;60:5565-5570.

31. Brekken C, Hjelstuen MH, Bruland ØS, de Lange Davies C. Hyaluronidase-induced periodic modulation of the interstitial fluid pressure increases selective antibody uptake in human osteosarcoma xenografts. Anticancer Res. 2000;20:3513-3519. 